\title{
Applications Analysis of Big Data Analysis in the Medical Industry
}

\author{
Zhendong Ji \\ Shandong Women's University, Shandong Jinan, 250300, China \\ jzd@sdwu.edu.cn
}

\begin{abstract}
With the promoting of big data analysis in every industry, the medical industry is also being widely used big data analysis as the tools, medical industry accumulated a large amount of data because of the wide ranging, it's has an important role in creating new business value for the medical industry and enhancing healthcare industry by performing the analysis of big data.. This paper focuses on the analysis of big data applications in the medical industry, and discussed the potential of its commercial value for the healthcare industry.
\end{abstract}

\section{Keywords: Big Data; Medical Industry; Business Value}

With the advent of the Internet era, economic social come into the analysis of big data era, almost all industries have begun to apply statistical analysis and forecasting by large data analysis, and big data analysis tools bring the hope for new business models and scientific development zone for medical industry. Using big data analysis in the medical industry, it's not only can enhance the commercial value of the medical industry, but also has an important reference value for medical research.

\section{Big Data in Medical Industry}

With the development in so many years, the medical industry has become a complete ecosystem, there are a lot of roles in this system, including medical institutions who provide the medical services, insurance companies who provide products or services of pay and government health or regulatory agencies who regulated at all levels of healthcare sector, as well as companies who production or research of drugs and medical equipment, etc., each role these systems provide data services to the public or consumer, thus the data obtained is very large, which constitute the big data in medical industry together, and the owner of these data are the various roles in healthcare ecosystem, they each have different types of big data, through these different types of systems conduct a comprehensive analysis of big data, and which has a great value for mastering the medical industry development situation and future trends.

Furthermore, the medical profession will produce large amounts of data in the actual operation, such as medical institutions will save a lot of cases the patient, including the patient personal information, disease and the consumption and other data, by analyzing of these data, it is not only can help parties understand the medical costs of medical conditions, but also to understand the development of diseases, which has important reference value for medical research. Medical management institutions at all levels can understand the epidemic situation occurred in all regions through the analysis of these data, at the same time can acquire all regional medical usage of resource, and thus can manage the medical profession more effective, to provide reference data for the Government to formulate policies in the management of the medical profession.

In terms of healthcare companies, medical enterprises requires large amounts of data to support the R \& D in medicine or medical device process, such as drug reaction testing, equipment application efficiency, etc., which are required on the basis of collecting a lot 
of data, this making healthcare companies are storing a lot of medical data, the companies can research and develop more advanced and humane medical products by the analysis of these data, thus contributing to the continuous improvement the medical standards. Thus, the big data of medical data have occupied an important position in various roles in the healthcare system, analyzing these big data has an important role to promote the healthy development of the ecosystem in the entire medical industry.

In the current situation of the medical industry, big data analysis tools is not very widespread applied in various roles in the healthcare industry, first is because the role of the medical profession is more, which result integrated analysis the different data of each role cannot be realized, and how to share the big data of medical industry ecosystem is a key issue. The second one is although Internet technology has been widely used in various industries, Internet technology and digital technology is still in the early development in the medical industry, a lot of medical data is not converted into electronic data, which makes more difficulty in the integration of these data and analysis, for example a lot of patients data are stored in paper form in hospital or clinical, and not converted into electronic files, which greatly inhibits the development of big data analysis tools applied in patient case data analysis. The third one is because medical information industry is still in an early stage of development currently, there are many IT providers, and medical institutions have an various of requirements in the information technology, which makes information technology cannot get healthy development, which indirect block the development of the technology of medical data analysis. Faced with these challenges, the health industry management department should strengthen its efforts to promote the development of data analysis techniques, integrate medical data in reason, and promote the applications of big data analysis in the medical industry.

\section{Big Data Analysis Applied in the Medical Industry}

\subsection{Big Data Analysis used in the Clinical Diagnosis}

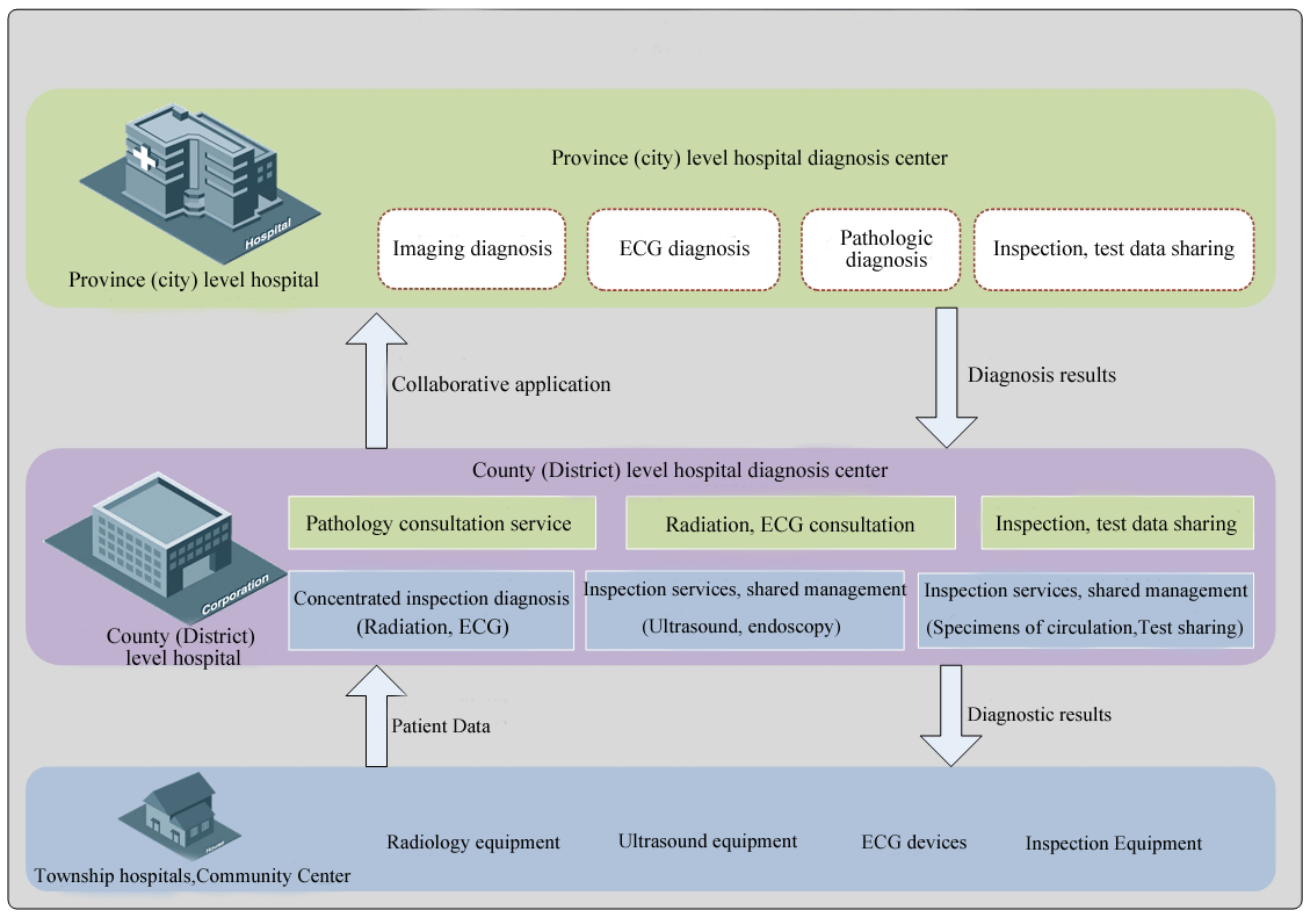

Figure 1. Hospital Inspection Diagnosis Support System 
The important application of big data analysis is to help clinical diagnosis in the healthcare industry, to provide data to support clinical policies and improve the level of clinical care. As shown in Figure 1,for the hospital inspection treatment support system. Clinical care is the first line of the healthcare industry, it is the most forward medical practitioners directly faced medical problems, and constantly improve the level of clinical care is the fundamental of the medical industry development, and enhance the level of clinical care often requires practitioners themselves have higher skills and learning ability, but a person's ability is limited, no matter how hard the medical staff could not break through the limitations of the individual, which is limited for the promotion of the overall medical standards development, the applications of the big data analysis in clinical care can be set in the wisdom of the crowd, breaking personal limitations and helping medical staff analyze more cases and clinical pathways, thus providing more effective diagnostic strategies or treatment programs for medical staff. By means of big data analysis can compensate for the lack of personal information acquisition and processing, but one can not be too dependent on big data analysis, which can just play a supporting role in the clinical care, after all, medical staff faced thousands of patients, each patient's condition is different and the medical staff should combine personal knowledge and experience with big data analysis in the actual operation.

Such as Angelina $\cdot$ Jolie, she carried the BRCA1 gene which may cause breast or ovarian cancer, she was given a series of preventive surgery and treatment to reduce the risk of cancer and improve the individual's health index on the basis of communicating with the doctor, in this process the effective tool testing gene sequencing is a kind of big data analysis tools in the process, so the big data analysis has already played an important role in clinical practice.

Table 1. Digital Hospital Construction EHR Modules

\begin{tabular}{|c|c|c|}
\hline System Name & Contains modules & The brief description of the module \\
\hline \multirow{8}{*}{$\begin{array}{l}\text { Electronic } \\
\text { medical record } \\
\text { system (EMR } \\
\text { ) }\end{array}$} & $\begin{array}{l}\text { Information retrieval } \\
\text { function module }\end{array}$ & $\begin{array}{l}\text { Through complaints, current medical record, } \\
\text { physical examination and other kinds of query } \\
\text { conditions to retrieve patient treatment records. }\end{array}$ \\
\hline & $\begin{array}{l}\text { Prescription } \\
\text { processing module }\end{array}$ & $\begin{array}{l}\text { Add, delete, and stop, save medical orders } \\
\text { processing functions of various orders. }\end{array}$ \\
\hline & $\begin{array}{l}\text { Medical writing } \\
\text { function modules }\end{array}$ & $\begin{array}{l}\text { Writing patient records by way of various types of } \\
\text { templates, quick and efficient. }\end{array}$ \\
\hline & $\begin{array}{l}\text { Care Information } \\
\text { Function Module }\end{array}$ & $\begin{array}{l}\text { Check the patient body temperature, blood } \\
\text { pressure, single record critically ill patients in } \\
\text { nursing records and other kinds of nursing } \\
\text { information. }\end{array}$ \\
\hline & $\begin{array}{l}\text { Electronic application } \\
\text { form functional } \\
\text { modules }\end{array}$ & $\begin{array}{l}\text { Inspection Requisition, check requisitions, } \\
\text { consultation and other electronic application form } \\
\text { to apply for a single use. }\end{array}$ \\
\hline & $\begin{array}{l}\text { Patient information } \\
\text { modules }\end{array}$ & $\begin{array}{l}\text { Check patient prescription information, testing } \\
\text { information, check the information, medical } \\
\text { information, medical care and information, and } \\
\text { other information. }\end{array}$ \\
\hline & History Query Mod & $\begin{array}{l}\text { Provides powerful query functions of the } \\
\text { historical record, including all previous medical } \\
\text { information. }\end{array}$ \\
\hline & The message to & Automatically to remind the quality control \\
\hline
\end{tabular}




\begin{tabular}{|c|c|}
\hline $\begin{array}{l}\text { remind function } \\
\text { module }\end{array}$ & information, crisis value information, vital signs. \\
\hline Record control system & $\begin{array}{l}\text { Control records of integrity, time, and improve the } \\
\text { quality of medical doctor writing. }\end{array}$ \\
\hline $\begin{array}{l}\text { Medical records } \\
\text { management system }\end{array}$ & $\begin{array}{l}\text { Medical record registration, borrowing, statistics, } \\
\text { filing of records management. }\end{array}$ \\
\hline $\begin{array}{l}\text { Remote medical } \\
\text { system }\end{array}$ & $\begin{array}{l}\text { Realization of remote viewing of patient records, } \\
\text { doctor's advice and other kinds of medical record, } \\
\text { can be used as a remote medical consultation } \\
\text { center. }\end{array}$ \\
\hline
\end{tabular}

The important way in clinical application of big data analysis is electronic medical records, as shown in table 1; this is the medical record system hospital construction according to the function module. electronic medical records currently has initially gained popularity in our country, to facilitate the use of big data analysis tools to integrate the personal information data and disease data converted into electronic data by electronic medical records, but the electronic medical records will reduce the efficiency of medical personnel and result the patients will be not satisfied with the medical staff job, thus how to combine the electronic medical records with medical staff to work more closely to improve healthcare efficiency becomes the important step on clinical applications.

While another broader clinical application tool of big data analysis is that clinical decision support systems, referred to CDSS, this system can combine the clinical data observed in the past with medical expertise to provide a variety of clinical select, thereby assist doctors make better clinical decision, the promotion of the system can improve the efficiency of doctors and improve the level of clinical treatment, so there are some experts thought that the future development terminal of electronic medical records system is CDSS.

Dating back in the 1970s, Stanford University has developed MYCIN system which have already closed with CDSS system, which is the oldest CDSS system and had a significant impact on the late CDSS, and the system can help diagnose severe bacterial infection and provide the appropriate antimicrobial drugs based on the patient's weight or illness, and then more and more CDSS system applied in subsequent clinical and involved in clinical decision-making, and combined more closely with clinical diagnosis and treatment, such as when do the examination of illness or physical for patient, these systems can take the initiative to remind doctors to provide the appropriate checks for the physician to more accurately determine the patient's condition. Such as the HELP system used in clinical Now, this system can be applied to the entire treatment process to assist with reminding and interpret data, and improve standard of the diagnosis and treatment. The system will automatically start when the patient's electronic medical record changes and provide diagnostic recommendations for doctors, when the patient's course of treatment carried to a new stage, the system can also provide the advice of treatment process for doctor to help medical staff to successfully implement the entire treatment process. 


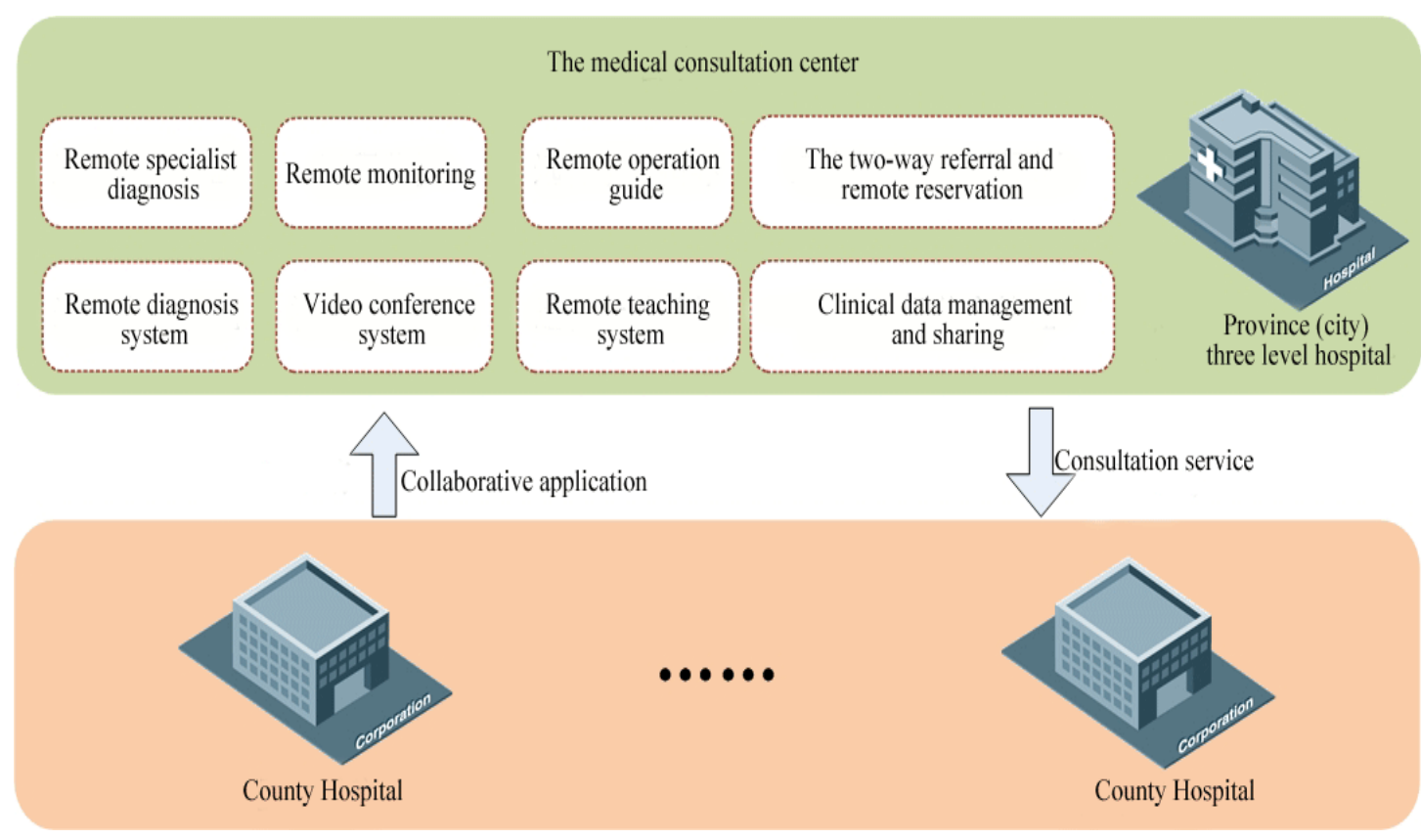

Figure 2. Remote Consultation System

In the future of the medical industry, the range of applications of CDSS system will also be more widely. As shown in Figure 2, this is a remote consultation system. which requires not only at the technical level to have a continuous breakthrough, but also in terms of user experience, as it relates to a very wide range and not only doctors and nurses, as well as other department staff, how to integrate these users data more closely with the system to provide better clinical decision support is the focus of future development of CDSS system. In addition, single disease CDSS system applied more in the current, how to broaden the kind of diseases of supported by the system and make the multiple diseases in one system is an important issue of the development of CDSS system.

\subsection{Big Data used in Personalized Service in Medical}

With the advanced medical concepts be absorbed and fusion continuously, the understanding of healthcare are also just not stop at the level of diagnosis and treatment, but more turned to health and emphasize the principle of treatment first before disease, for which produces personalized healthcare service also gradually become the main force in the medical industry. Big data analysis received widely attention in personalized healthcare services, patients can be provided with a more humane and personalized treatment, and integrated and analyzed health factors is an effectively way to prevent the occurrence of disease, which has an important significance in promotion of the level of the whole community residents' health. Specifically, the big data analysis applied in personalized medicine services mainly contained in the following aspects:

Firstly, personalized medicine service. Through analyzing the medical big data to investigate the relationship between genetic variation, a special drug reactions and susceptibility of specific diseases, and then use the results to drug research, which will be able to produce a richer personalized medicine to improve the therapeutic effect of drugs and reduce drug reactions. a lot of the patient's condition is the same usually, but with the same treatment the effect obtained is not the same, this is because each person has different physical, genetic, and in order to reduce the difference and reduce the impact of genetic factors effecting on drug efficacy, for different patients should be given different treatment options, and should be adjusted drugs according to the patient's condition to reduce drug side effects and reactions and improve drug treatment, big data analysis 
played a very important role to assist in the process, through big data analysis can understand the genetic factors that influence the proportion of drug efficacy, the analysis of susceptible populations and provide personalized care by treatment intently. For example Jobs applied the big data analysis techniques when undergoing cancer treatment, using DNA sequencing to sort his own genes, so that doctors can issue the appropriate medication according to his specific genes, so this can have an effect of the therapeutic target, Once a drug fails, doctors can replace drugs according to his genes, the personalized medicine can prolong the life of Steve Jobs and bring hope to cancer treatment in the future.

Secondly, gene sequencing services. Angelina Jolie above-mentioned she adopted prevention treatment measures under the circumstances of illness genes carried by herself, she is the application of gene sequencing services to learn the gene illness herself carried, which is one of the most important applications of personalized medicine by the big data analysis. Gene sequencing has begun to be promoted currently in the United States, it is applied to influenza control in many parts, but with the development of science and technology, the cost of DNA sequencing has become increasingly low, so that more ordinary people can enjoy the gene sequencing services. Currently the United States has more than 2000 gene sequencing companies offers services, there will be more and more companies offer this service in the future, which means that people will be able to develop personalized healthcare program based on their gene sequencing in future Through gene sequencing to integrate gene database analysis deeply and understand the role of genes in the development of disease, detecting of disease information that exist in the genes, and it is the genes information that leads to such rare diseases as the neonatal disease, cancer and sickle cell anemia. These data analysis will be able to provide the most basic research material for research institutions, medical staff and other healthcare providers to help medical assistance or research institutions to provide clinical data supporting of medical research, promote and improve diagnosis and treatment levels of the medical battlefront. And in the United States in order to implement personalized medicine services, in the coming years Wisconsin Medical Center combined with Bina scientific and technological cooperation do gene sequencing for children in neonatal intensive care unit, and hope that the birth of the newborn are able to accept gene sequencing for providing the big data base to neonatal care, to provide data for analyzing neonatal intensive. With the technology company's platform, medical teams can complete hundreds of newborns gene sequencing analysis in just five hours and gain a lot of genetic which offer more possibilities information for the future development of medical research. It is foreseeable that in the future gene sequencing will have very big impact on our health, it is possible to change the way of seeing the doctor in future and also reveal the advent of personalized medicine.

Thirdly, personal health management services. Big data analysis technology can provide a full range of personal health management with the whole process, personal health information can be accessed at any place or time, which allows individuals to keep abreast of their health situation and adjustment timely. There are a lot of companies have begun to offer this kind of management services, such as Microsoft's Health Vault platform, and Google Health service provided by Google, and even with the introduction of smart phones, a lot of health management software are applied to the smart phone, so individuals are capable of continuous observation of their own health status, and thus the effective analysis of their own health situation and make the body can be in the good health condition in the long-term, and intervention non-health status timely, which undoubtedly can improve individual health and prolong human lifetime. In the past health management, the individual detect abnormal health through periodic medical examinations and take appropriate interventions, but this management style is separated too long, it is impossible to do a medical examination every day, individual cannot be found and treated when the disease or risk factors occurred and leads to delays healing, 
affecting the health of the individual. It is possible to monitor the individual health in time by means of individual health management facilities, monitoring the individual's body data, such as heart rate, blood pressure, body temperature, respiratory rate, blood glucose, oxygen, BMI index, to keep abreast of individual changes, in the characteristics of the data, which can timely detect the disease or hidden risk factors and treat promptly, and effectively safeguard the health of individuals. Currently the body data measurement tools available in the market are mainly including temperature sensor, weight measurement sensors, heat flux sensor, pulse sensor, bio-electric sensors, and optical sensors, etc., these devices will be timed on human data to automatically detect signs throughout the day, in order to establish personal health management model, using the big data analysis techniques will be able to carry out further analysis of these data, formatting the healthrelated reports to allowing users to understand their own health, understand their health ranking in the same age or gender, improve health according to the ranking take timely measures to intervene. And big data analysis tools can also adjust themselves, once detected the abnormal data of the individual, the detect density increases also, grasp the individual signs data changes, if the individual data properly, it will be appropriate to increase the detection interval to achieve dynamic detection. Personalized health management tools has been promoted in a certain amount, and in the future of personalized medicine will become the core of the service.

\section{Some Advices about the Application of Big Data Analysis in Medical Industry}

Because of the rapidly changing network environment and the existence of data information security problem, as well as the characteristics of big data, such as large quantity, wide range, multi levels and low-density of magnitude of value, at the same time, due to the late start of big data analysis processing technology in China, especially in the medical industry, deep research and exploration are needed. Therefore, some advices about the application of big data analysis in medical industry are put forward:

\subsection{Promote the Effective Recognition of Big Data Analysis}

There is much repeated, invalid and false information in collecting and sorting data; in addition, the network data itself is subjective, especially information on micro-blog, forum and other social networks, with much private emotion, which leads to great information uncertainty. Based on this, we should pay attention to the conditional filtering of information in collecting and storing data information. For example, for medical industry, due to the differences between actual technology and service of medical personnel and other personnel and consumer expectations, consumers will have poor motives psychologically. The information they issued on the Internet is likely to be with much personal prejudice and private emotion, subjective. Or alternatively, consumers spread their dissatisfaction with medical personnel caused by their personal reasons to the whole enterprise, even the whole industry, which is a deviation of the actual situation. Therefore, we should focus on the discrimination of data in collecting and sorting network data, especially the recognition of some extreme information.

\subsection{Broaden the Collection Channel and Scope of Big Data}

One of the biggest characteristics of network data is the large base, wide range and multi levels. The first premise for big data analysis is to obtain certain valid data information. For the data analysis in the medical industry, in order to obtain accurate and real data information, we must take two aspects into consideration: one is the medical industry's point of view, and two is the consumer perspective. Only by combining the two aspects together, can we effectively compensate for the deviation of data information 
collected through different ways, so as to ensure the veracity of data information. Moreover, when collecting data information related to consumers, we should divide consumers into different levels, such as white-collar workers, public officials, teachers, workers and so on, and conduct multilevel and wide-range data information collection; when collecting data information related to medical enterprises, we should pay attention to relevant data information on enterprise's website and official data information issued by government departments, thus improving the validity and accuracy of data.

\subsection{Time Limit Grasp of Data Collection and Data Analysis}

Big data analysis has high requirements on the time limit, so it is required to grasp the time limit in collecting, sorting and analyzing data. The current network data seems like stock information and the change of rising and falling is so quick that the information that is useful at this moment may be invalid next moment or that late collection and analysis will allow other enterprises to seize the opportunities. Therefore, the units involved in the medical industry should pay more attention to the market trend, holding the chance and obtaining the data value, so as to win the initiative in market competition.

\section{Conclusion}

In summary, the applications of big data analysis in the medical industry will be more and more widely in the future, everyone engaging in the medical accumulated provides an important condition for big data analysis in future, it has an great value for prompted the trends for future and promoting the continuous development of the medical industry by meta-analysis of these data. Meanwhile, many of the current problems facing the healthcare industry can be resolved with a large data analysis in future, which provides significant value for continued development of the medical ecosystem.

\section{References}

[1] J. Xin and W. Jiaxin, "Big Data used in intelligent medical system construction [J]", Science and Technology Aspect of China, vol.2, no.14, (2014), pp.44-44.

[2] C. Pu, "Development of China's medical information under the big data environments [J]",Management theorists (Academic Edition), vol.3, no.6, (2014), pp.2-3.

[3] S. Xiyi and Y. Liqiang, "The changes the era of big data has brought to the medical information system development[J]", Technology Vision, vol.11, no.17, (2014), pp.313-314.

[4] L. Zhiqiang, K. Lijun and W. Wencui, "big data security management strategy research oriented to medical information[J]", Computer Security, vol.4, no.4, (2014), pp.84-86.

[5] Z. Jie, "Subversion of the traditional: mHealth revolution - subversion Medical: personal health revolution in the era of Big Data [J]", Health Management, vol.7, no.4, (2014), pp.11-13.

[6] W. HanHua, "How to do mining and utilization of medical data in big data era [J]", The Silicon Valley, vol.7, no.5, (2014), pp.13-13.

[7] L. Jia, "EHR assessment in grading: Tackling Big Data Challenge [J]", Hospital lead the decisionmaking reference, vol.5, no.1, (2014), pp.37-38.

[8] L. Yating, "Big Data promote medical wisdom landing [J]", Economy and information of China, vol.2, no.7, (2014), pp.90-91.

[9] W. Sheng and L. Yajun, "Medical thinking in the era of big data[J]", China Book Reviews, vol.1, no.3, (2014), pp.125-128.

[10] L. Jing, "The opportunities and challenges of personalized medicine and the era of big data [J]", Medicine and Philosophy (Humanities and Social Medicine Edition), vol.10, no.1, (2014), pp.5-10.

[11] C. Pu, "The problems and countermeasures of medical information development in Big Data era [J]", China Electronic Business (Technological and Innovation), vol.11, no.23, (2013), pp.49-49.

[12] Z. Beiji, "Big Data analysis and its applications in the field of medical [J]", Computer Education, vol.11, no.7, (2014), pp.24-29. 


\section{Author}

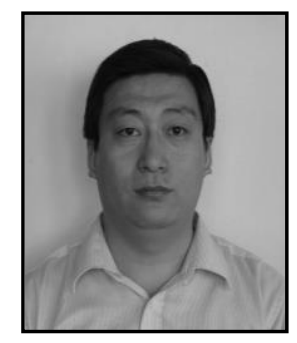

Zhengdong Ji, received the B. Sc in Applied Mathematics from Shandong Normal University (SDNU) and M. Eng degree in Software Engineering from Shandong University (SDU) in 2000 and 2011respectively. He is currently researching on Data Mining (DM) and Mathematical Modeling.. 
International Journal of Database Theory and Application Vol.8, No.4 (2015) 